\title{
Repulsive gravitational effect of a quantum wave packet and experimental scheme with superfluid helium
}

\author{
Hongwei Xiong ${ }^{1,2}$ \\ ${ }^{1}$ Wilczek Quantum Center, Zhejiang University of Technology, Hangzhou 310023, China \\ ${ }^{2}$ College of Science, Zhejiang University of Technology, Hangzhou 310023, China \\ Corresponding author. E-mail: xionghw@zjut.edu.cn \\ Received April 21, 2015; accepted May 14, 2015
}

\begin{abstract}
We consider the gravitational effect of quantum wave packets when quantum mechanics, gravity, and thermodynamics are simultaneously considered. Under the assumption of a thermodynamic origin of gravity, we propose a general equation to describe the gravitational effect of quantum wave packets. In the classical limit, this equation agrees with Newton's law of gravitation. For quantum wave packets, however, it predicts a repulsive gravitational effect. We propose an experimental scheme using superfluid helium to test this repulsive gravitational effect. Our studies show that, with present technology such as superconducting gravimetry and cold atom interferometry, tests of the repulsive gravitational effect for superfluid helium are within experimental reach.
\end{abstract}

Keywords gravitational effect of quantum wave packet, precision measurement, cold atoms

PACS numbers 04.60.Bc, 04.80.Cc, 05.70.-a

\section{Introduction}

Although the unification of quantum mechanics and general relativity is elusive, considerable theoretical studies to reveal possible macroscopic quantum gravitational effect have been presented. A classic example is Hawking radiation [1], predicted by combining general relativity, quantum mechanics, and thermodynamics. Although the quantum gravitational problem is far from solved, various approaches are being used to find evidence of quantum gravitational effects in high-energy scattering experiments and astronomical observations [2,3]. Many novel ideas are also proposed to test the quantum gravitational effects, e.g., probing the Planck-scale physics with a mechanical oscillator [4], the search for dark energy using atom interferometry [5], and the measurement of homological noise [6, 7]. At present, no evidence of quantum gravitational effects have been observed in experiments on Earth.

In this study, we consider the gravitational effects for a quantum wave packet based on the thermodynamic origin of gravity [8]. By considering the fact that the direction of force can be naturally interpreted in thermodynamics, we find some justification for the attractive characteristics of Newton's law of gravitation. With the same considerations, we discuss a possible repulsive grav- itational effect for quantum wave packets. It is clear that, without a well-defined solution to the quantum gravitational problem at the Planck length, this phenomenological theory requires experimental testing. Fortunately, our studies show that current techniques for measuring the gravitational force such as superconducting gravimetry and cold atom interferometry are able to test repulsive gravitational effects in superfluid helium.

The paper is organized as follows. In Section 2, we provide an explanation of the attractive gravitational force in Newton's law of gravitation. Section 3 is devoted to the consideration of the gravitational effects for a quantum wave packet when both quantum mechanics and thermodynamics are considered. We present a general equation for the gravitational force for a quantum wave packet. In Section 4, using the general equation presented in the third section, we propose an experimental scheme to test the repulsive gravitational effect for superfluid helium. A brief summary and discussion are presented in the final section.

\section{Thermodynamic understanding of the direction of gravitational force in Newton's law of gravitation}

It is well known that the law of gravity closely resem- 
bles the laws of thermodynamics and hydrodynamics [914]. This has led to intensive studies [15-26] into the thermodynamic origin of gravitation [8]. When the thermodynamics of a system are considered, the direction of the force (e.g., pressure) can be determined from the thermodynamic properties of the system. This leads to a question about whether there is a physical mechanism determining the direction of the gravitational force, if the thermodynamic origin of gravitation is assumed. In this section, we provide possible answers to this question.

Recently, the thermodynamic understanding of gravitation has been significantly advanced by Verlinde's work [27], in which the change of entropy $S$ after a displacement $x$ is given by the following formula:

$$
S=2 \pi k_{\mathrm{B}} \frac{m c}{\hbar} x .
$$

Here, $m$ is the mass of a fundamental particle, $c$ is the speed of light, and $k_{\mathrm{B}}$ is the Boltzmann constant. In the original work by Verlinde, this postulation (motivated by Bekenstein's work [9] regarding black holes and entropy) plays a key role in deriving Newton's law of gravitation. The proportional relation between $S$ and $x$ can be partially explained by an entropy increase with information loss. For a particle's motion in the vacuum background, when the particle arrives at a location after a displacement $x$, the information regarding its path is lost, which leads to an entropy increase when the vacuum background is also included.

Although there is significant controversy regarding the meaning and validity of this thermodynamic formula, it deserves further study. Considering a particle with acceleration $\boldsymbol{a}$, we have

$$
x_{j}=\frac{a_{j} t^{2}}{2} .
$$

Here, $j=1,2,3$. In this paper, all bold symbols represent vectors. From Eq. (1), we have

$$
\mathrm{d} S=\frac{2 \pi k_{\mathrm{B}} m c}{\hbar} \sqrt{\sum_{j}\left(a_{j} t\right)^{2}} \mathrm{~d} t .
$$

In addition, from $E=\sqrt{m^{2} c^{4}+p^{2} c^{2}}$, using the nonrelativistic approximation, we have

$$
\mathrm{d} E=m \sum_{j} a_{j}^{2} t \mathrm{~d} t
$$

Using the fundamental thermodynamic relation $\mathrm{d} E=$ $T_{V} \mathrm{~d} S$, we have

$$
T_{V}=\frac{\hbar}{2 \pi k_{\mathrm{B}} c} \frac{\sum_{j} a_{j}^{2}}{\sqrt{\sum_{j} a_{j}^{2}}}=\frac{\hbar|\boldsymbol{a}|}{2 \pi k_{\mathrm{B}} c} .
$$

It is clear that this temperature $T_{V}$ is different from the ordinary temperature for an ensemble of particles in thermal equilibrium. In this acceleration process, there is no entropy increase for the particle itself. In the acceleration process of the particle, the only possibility for entropy increase comes from the vacuum, if Eq. (1) is correct. Thus, $T_{V}$ refers to the vacuum temperature at the location of the particle. This shows that the acceleration of a particle will induce vacuum excitations, and thus, lead to finite vacuum temperature at the location of the particle. Because there is a decay of these vacuum excitations in the propagation process, $T_{V}$ should be a spatially dependent temperature field distribution with a maximum value given by $\hbar|\boldsymbol{a}| /\left(2 \pi k_{\mathrm{B}} c\right)$. As we do not have exact knowledge at the Planck length, it is not within the scope of the present work to provide the exact spatial scale of the decay. However, at least in the present work, the maximum value of $T_{V}$ is the physical quantity we need.

It is noteworthy that the above equation is the same as the Unruh temperature [13]. This indicates the selfconsistency of the above derivation. In Verlinde's work, both Eqs. (1) and (5) are used to obtain Newton's law of gravitation. Here, we show the possibility of further simplification because Eq. (5) can be derived from Eq. (1).

The meaning of Eq. (5) is further explained by Fig. 1. For a particle at location B with acceleration $\boldsymbol{a}_{\mathrm{B}}$ (shown by the red arrow), the coupling between the particle and the vacuum modes establishes the vacuum temperature field distribution (shown by the dashed blue line) with a peak value of $T_{V}\left(\boldsymbol{a}_{\mathrm{B}}\right)=\hbar\left|\boldsymbol{a}_{\mathrm{B}}\right| /\left(2 \pi k_{\mathrm{B}} c\right)$. The strong coupling between the particle and the vacuum modes leads to a "dressed" state that includes the local vacuum excitations and the particle itself. If the particle has no size, roughly speaking, the width of the local vacuum excitations is of the order of the Planck length (using the same treatment for the derivation of Newton's law of gravitation used in the latter part of this section).

Eq. (5) shows the vacuum temperature field due to an accelerating particle. It is natural to consider the opposite problem: What is the acceleration of a particle in the presence of a finite vacuum temperature field distribution? In Fig. 1, we assume that there is a vacuum temperature field distribution (shown by the red line) that is due to a system denoted by A, e.g., a celestial body. At location B, there is a particle (denoted by a red sphere). To establish local thermal equilibrium, the red sphere will accelerate such that the peak vacuum temperature of the dressed state is equal to the temperature of the vacuum temperature field at location $\mathrm{B}$, i.e., $T_{V}\left(\boldsymbol{a}_{\mathrm{B}}\right)=T_{V}(\mathrm{~B})$. In this situation, we have 


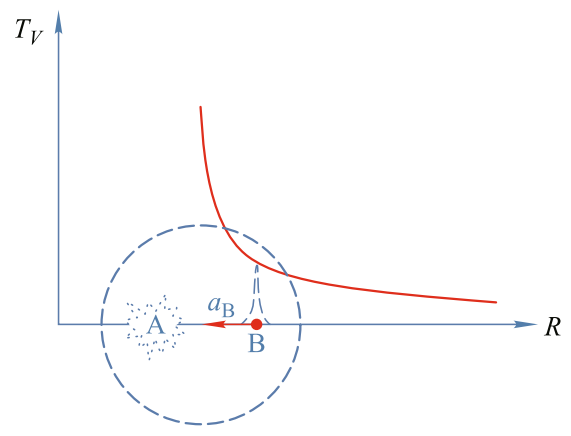

Fig. 1 The relation between the vacuum temperature field distribution and acceleration. The red line shows the vacuum temperature field distribution for a system denoted by A, e.g., a celestial body. The red sphere represents an initially at rest classical particle at a location B. The acceleration $\boldsymbol{a}_{\mathrm{B}}$ of particle $\mathrm{B}$ induces a vacuum temperature field distribution (indicated by the dashed blue line) with a peak value given by Eq. (6). The local thermal equilibrium requests that this peak value is equal to the temperature of the vacuum temperature field (red line) at location B. This shows the physical mechanism that causes particle B to accelerate in the presence of a finite vacuum temperature field. For the whole system (surrounded by the dashed blue circle), the application of the free energy in the canonical ensemble determines the direction of the acceleration. With this consideration, the direction of the acceleration is determined by the gradient of the vacuum temperature field distribution, indicated by the red arrow.

$$
\left|\boldsymbol{a}_{\mathrm{B}}\right|=\frac{2 \pi k_{\mathrm{B}} c T_{V}(\mathrm{~B})}{\hbar} .
$$

However, the acceleration is a vector, whereas the temperature is a scalar. Therefore, the above formula does not define the direction of the acceleration. For the whole system (including system A and particle B surrounded by the dashed blue circle), it is clear that the particle number is conserved while there is energy exchange, which is due to the propagation of the vacuum excitations. In this case, the canonical ensemble should be used in thermodynamic studies of the whole system. Therefore, we consider the free energy of the whole system, which is defined as $F=U-T_{V} S$. Here, $U$ is the overall energy of the system, which is a conserved quantity. Obviously, the whole system is a non-equilibrium system. In thermodynamics, it is well established that system evolution has a tendency to decrease the free energy in the most effective way. Under this consideration, we use the following formula to determine the magnitude and direction of the acceleration in the presence of a vacuum temperature field:

$$
\boldsymbol{a}(\boldsymbol{R})=\frac{2 \pi k_{\mathrm{B}} c T_{V}(\boldsymbol{R})}{\hbar} \frac{\nabla_{\boldsymbol{R}} T_{V}(\boldsymbol{R})}{\left|\nabla_{\boldsymbol{R}} T_{V}(\boldsymbol{R})\right|} .
$$

Here, $\boldsymbol{R}$ denotes a three-dimensional spatial vector, and $\nabla_{\boldsymbol{R}}$ denotes the vector differential operator. In Fig. 1, for a particle at location $\mathrm{B}$, the direction of the acceleration is based on the consideration of the free energy. We will show that the above equation provides an explanation of why the gravitational force is attractive between two spatially separated objects.

Now, we consider Newton's law of gravitation under the assumption of the thermodynamic origin of gravitation. In Fig. 2, we consider a space with a Planck length $l_{p} \equiv \sqrt{\hbar G / c^{3}}$, which shows the structure of the phase space due to the quantum gravitational effect. Here, $G$ is the gravitational constant. Although the microscopic mechanisms of $l_{p}$ are not completely clear, it is not unreasonable to assume the existence of $l_{p}$ in the phase space. We assume that the energy of a particle (indicated by a red sphere in Fig. 2) is $\varepsilon$. We further assume that the number of degrees of freedom is $i$ for the elementary cells. From the local thermal equilibrium, at the location of the particle, the temperature of the vacuum is given by

$$
\frac{\mathrm{i}}{2} k_{\mathrm{B}} T_{V}\left(R<l_{p}\right)=\gamma \varepsilon .
$$

Here, $\gamma$ denotes a dimensionless coupling strength between matter and space. From ordinary statistical mechanics, $\gamma$ should be of the order of 1 . We have

$$
T_{V}\left(R<l_{p}\right)=\frac{2 \gamma \varepsilon}{\mathrm{i} k_{\mathrm{B}}} \text {. }
$$

When $R \rightarrow \infty, T_{V}=0$. Thus, one expects the vacuum temperature field distribution shown in Fig. 2. For another particle with mass $m$ in this vacuum temperature field distribution, from Eq. (7), the acceleration field distribution is then

$$
\boldsymbol{a}=-\frac{2 \pi k_{\mathrm{B}} c T_{V}}{\hbar} \boldsymbol{e}_{R}
$$

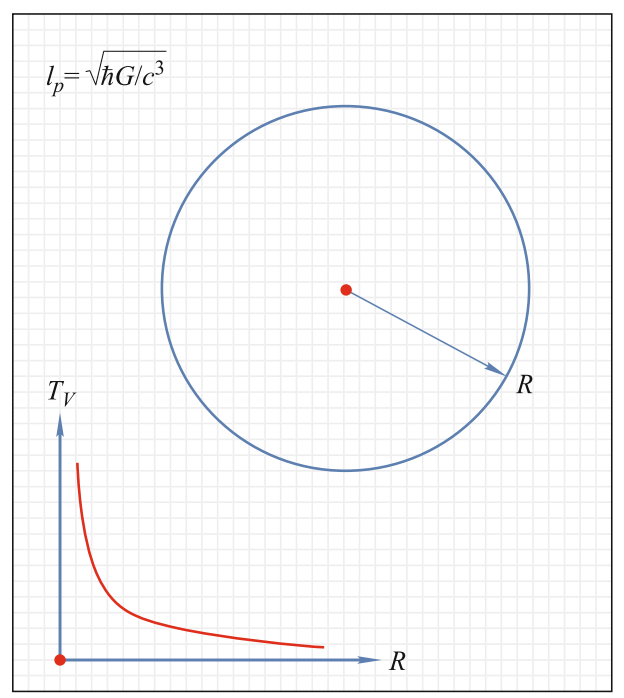

Fig. 2 The grid shows the Planck length due to the quantum gravitational effect. The red sphere shows a particle that leads to a vacuum temperature field distribution through coupling with the vacuum. The vacuum temperature field distribution is also shown in this figure. 
Here, the radial unit vector $\boldsymbol{e}_{R} \equiv \boldsymbol{R} /|\boldsymbol{R}|$. The negative sign in the above equation derives from both the gradient of $T_{V}(\boldsymbol{R})$ in the relation between $\boldsymbol{a}$ and $T_{V}(\boldsymbol{R})$ and the relation in which a gradual decrease in $R$ results in an increase in $T_{V}(\boldsymbol{R})$. To obtain the above expression, the spherical symmetry of the system for $R \gg l_{p}$ is also used. This equation explains the attractive gravitational force between two classical objects. It is worth pointing out that in Newton's law of gravitation, the attractive gravitational force is based on observations, rather than microscopic mechanisms. One of the merits of thermodynamics is that even if we do not know the exact collision properties (such as the scattering length between atoms), the macroscopic forces (such as pressure) can be derived. When the thermodynamic origin of gravity is assumed, we should expect the correct result for the direction of the gravitational force.

The continuous property of the gravitational force adds a further constraint on the asymptotic behavior of the vacuum temperature field distribution for $R \gg l_{p}$. For this reason, we have

$$
T_{V}(\boldsymbol{R})=\frac{\eta}{R^{2}} .
$$

Note that the above expression holds for $R \gg l_{p}$; therefore, the spherical symmetry approximation can be used.

Combined with Eq. (9), we have $\eta / l_{p}^{2}=2 \beta \gamma \varepsilon /\left(\mathrm{i} k_{\mathrm{B}}\right)$. We have introduced a factor $\beta$ to provide a more rigorous derivation. Although we do not know the exact value of $\beta$, the asymptotic behavior of $T_{V}$ indicates that $\beta$ is of the order of 1 . In this situation, we have

$$
T_{V}(\boldsymbol{R})=\frac{2 \beta \gamma M c^{2} l_{p}^{2}}{\mathrm{i} k_{\mathrm{B}}} \frac{1}{R^{2}} .
$$

To get the above expression, we used $\varepsilon=M c^{2}$. Using Eq. (10), we have

$$
\boldsymbol{a}=-\frac{4 \pi \beta \gamma c^{3} l_{p}^{2}}{\mathrm{i} \hbar} \frac{M}{R^{2}} \boldsymbol{e}_{R}
$$

Because the gravitational constant $G$ is a physical constant obtained from observations, the factor $4 \pi \beta \gamma / \mathrm{i}$ can be absorbed into it. Finally, we obtain the standard result of Newton's gravitation law

$$
\boldsymbol{a}=-\frac{G M}{R^{2}} \boldsymbol{e}_{R}
$$

We consider above-described the situation for a particle whose size is of the order of the Planck length. If the size of the particle is larger than the Planck length, using the Gauss's flux theorem for $R$ much larger than the size of the particle, the above result still holds. Without an exact theory at the Planck scale, it is obvious that the above derivation of Newton's law of gravitation is not a rigorous derivation from first principles. Nevertheless, we have some evidence of the physical mechanism of the attractive gravitational force in Newton's law of gravitation. In the next section, this idea will be applied to study the gravitational force of quantum wave packets.

For an assembly of classical fundamental particles (here "classical" means that the quantum wave packet effect is negligible), we assume that the vacuum temperature field distribution due to the $i$ th particle is $T_{V i}(\boldsymbol{R})$. Because there is no quantum interference effect between different classical particles, the force on an object with mass $m$ is

$$
\frac{\boldsymbol{F}(\boldsymbol{R})}{m}=\boldsymbol{a}(\boldsymbol{R})=\frac{2 \pi k_{\mathrm{B}} c}{\hbar} \sum_{i} \frac{T_{V i}(\boldsymbol{R}) \nabla_{\boldsymbol{R}} T_{V i}(\boldsymbol{R})}{\left|\nabla_{\boldsymbol{R}} T_{V i}(\boldsymbol{R})\right|} .
$$

The above expression is based on the assumption of the linear superposition of gravitational forces $\boldsymbol{F}=\boldsymbol{\Sigma}_{i} \boldsymbol{F}_{i}$. In Newton's law of gravitation for an assembly of classical particles, this implicit assumption is also used. We stress here that, rigorously speaking, the summation in the above expression is about all fundamental particles.

\section{Abnormal gravitational effect for a quantum wave packet}

In the thermodynamic origin of gravity, for an object with mass $M$, it establishes a vacuum temperature field $T_{V}(\boldsymbol{R}) \sim M /|\boldsymbol{R}|^{2}$. Using the formula $\boldsymbol{a}=$ $2 \pi k_{\mathrm{B}} c T_{V} \nabla_{\boldsymbol{R}} T_{V} / \hbar\left|\nabla_{\boldsymbol{R}} T_{V}\right|$ for the relation between the acceleration and vacuum temperature, we obtain Newton's law of gravity $\boldsymbol{F}=-G M m \boldsymbol{R} / R^{3}$ between two classical objects. In this section, we will consider the gravitational force when including the quantum wave packet effect in the thermodynamic origin of gravity.

We first consider the following fictional wave function for a fundamental particle with mass $m_{q}$,

$$
\begin{array}{ll}
\phi_{q}(\boldsymbol{x}, t) \simeq \frac{1}{\sqrt{V}} \quad\left(R<R_{0}\right), \\
\phi_{q}(\boldsymbol{x}, t) \simeq 0 \quad\left(R>R_{0}\right) .
\end{array}
$$

The average density distribution $\left|\phi_{q}(\boldsymbol{x}, t)\right|^{2}$ is indicated by the black quantum sphere in Fig. 3(a). Although this is a fictional wave function, its simple form contributes to our understanding of the abnormal gravitational effect for quantum wave packets.

For $R>R_{0}$, similar to the consideration of a classical particle, it is easy to obtain

$$
T_{V q}=\frac{\hbar G m_{q}}{2 \pi k_{\mathrm{B}} c R^{2}} .
$$

At $R=0$, based on the spherical symmetry, we have 
(a)

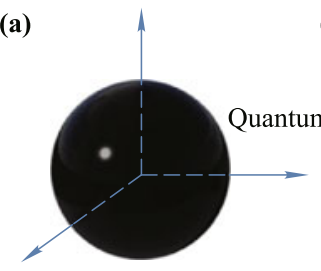

(b)

(c)

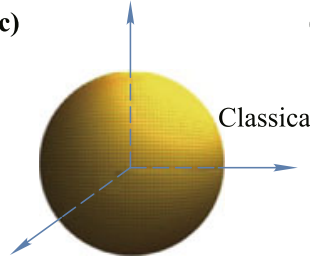

(d)

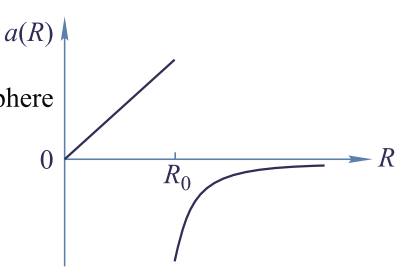

d) $a(R)$

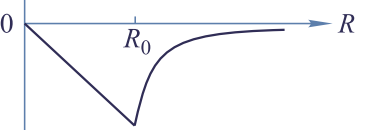

Fig. 3 (a) shows a wave packet distribution of a particle in the black sphere. (b) shows the gravitational acceleration of this quantum wave packet. (c) and (d) show the classical situation.

$\boldsymbol{a}(R=0)=\mathbf{0}$. Thus, based on the relation between $\boldsymbol{a}$ and $T_{V q}$, we have $T_{V q}(R=0)=0$. Another influence that suggests that $T_{V q}=0$ at $R=0$ is the interference effect of a wave packet with spherical symmetry. For $R<R_{0}$, again using the spherical symmetry and Gauss's flux theorem, we have

$$
T_{V q}=\frac{\hbar G m_{q} R}{2 \pi k_{\mathrm{B}} c R_{0}^{3}} \text {. }
$$

The whole vacuum temperature field distribution is shown in Fig. 4. We see that, in the interior of the quantum sphere, the vacuum temperature increases with increasing $R$. This agrees with the physical intuition that the absolute value of the acceleration field increases with increasing $R$ and becomes zero at $R=0$. In fact, based on the idea presented in the previous section regarding the origin of the gravitational force, the above equation is the only choice for the vacuum temperature field for the quantum sphere shown in Fig. 3(a).

For the quantum wave packet shown in Fig. 3(a), from the relation between the acceleration field and the vacuum temperature field distribution given by Eq. (7), we

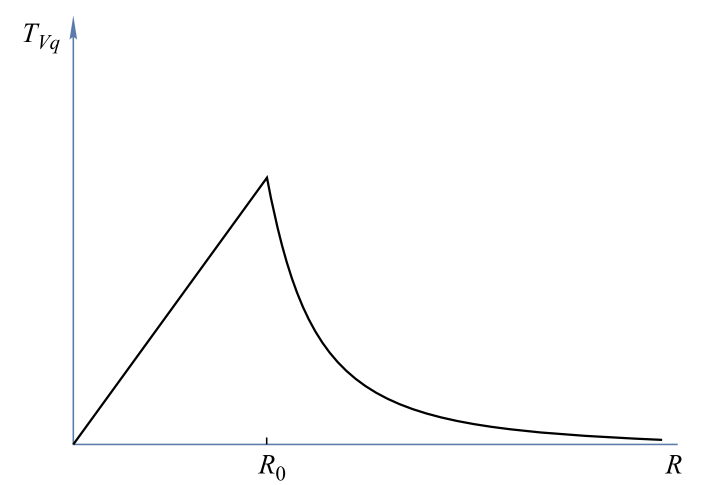

Fig. 4 The vacuum temperature field distribution for a particle with a wave function given by Eq. (16). have

$$
\begin{array}{ll}
\boldsymbol{a}=\frac{G m_{q} R}{R_{0}^{3}} \boldsymbol{e}_{R} & \left(R<R_{0}\right), \\
\boldsymbol{a}=-\frac{G m_{q}}{R^{2}} \boldsymbol{e}_{R} & \left(R>R_{0}\right) .
\end{array}
$$

This relation indicates that in the interior of the quantum sphere, the gravitational force is repulsive. This abnormal gravitational effect is further shown in Fig. 3(b). It is clear that this abnormal gravitational effect physically originates from the quantum wave packet effect for a particle in the black sphere. This phenomenon is reminiscent of the repulsive gravitational effect of dark energy. The application of this repulsive gravitational effect to dark energy [28-30] and the extension to general relativity will be given elsewhere.

Because the temperature field distribution in Fig. 4 is not continuous for the first-order derivative of $R$ at $R_{0}$, there is a discontinuity at $R_{0}$ in the direction of the acceleration field shown in Fig. 3(b) for the gravitational force because we are considering a quantum wave packet. Because, in the calculation of the temperature field, the spatial scale of the phase space is roughly the Planck length, it is expected that the discontinuous region would be of the order of the Planck length. In real-world situations, the quantum wave packet cannot be distributed as shown in Fig. 3(a) in an ideal way. Thus, the discontinuous region will be more complex than the ideal symmetric distribution of the quantum wave packet. The discontinuous distribution of the gravitational field provides a possible way for experimental tests.

If there are $N$ particles in the same quantum state given by Eq. (16), we have

$$
\begin{aligned}
\boldsymbol{a} & =\frac{2 \pi k_{\mathrm{B}} c}{\hbar} \sum_{i} \frac{T_{V i}(\boldsymbol{R}) \nabla_{\boldsymbol{R}} T_{V i}(\boldsymbol{R})}{\left|\nabla_{\boldsymbol{R}} T_{V i}(\boldsymbol{R})\right|} \\
& =\frac{G N m_{q} R}{R_{0}^{3}} e_{R} \quad\left(R<R_{0}\right), \\
\boldsymbol{a} & =\frac{2 \pi k_{\mathrm{B}} c}{\hbar} \sum_{i} \frac{T_{V i}(\boldsymbol{R}) \nabla_{\boldsymbol{R}} T_{V i}(\boldsymbol{R})}{\left|\nabla_{\boldsymbol{R}} T_{V i}(\boldsymbol{R})\right|} \\
& =-\frac{G N m_{q}}{R^{2}} e_{R} \quad\left(R>R_{0}\right) .
\end{aligned}
$$

To further understand the abnormal gravitational effect, we consider a classical sphere with the following density distribution of the mass

$$
\begin{aligned}
& n(\boldsymbol{x}, t) \simeq \frac{N m_{q}}{V} \quad\left(R<R_{0}\right), \\
& n(\boldsymbol{x}, t) \simeq 0 \quad\left(R>R_{0}\right) .
\end{aligned}
$$

This density distribution for a classical sphere is shown in Fig. 3(c). It appears that the same acceleration field 
distribution as that of the quantum sphere is found; however, this conclusion is not accurate. For a classical sphere, assuming there are $N$ particles, the wave packets of all particles are highly localized. Thus, for a particle at location $\boldsymbol{x}_{j}$, the vacuum temperature field distribution due to this particle is $T_{V j}(\boldsymbol{R}) \sim m_{j} /\left|\boldsymbol{R}-\boldsymbol{x}_{j}\right|^{2}$. From $\boldsymbol{a} \sim \Sigma_{j} T_{V j} \nabla T_{V j} /\left|\nabla T_{V j}\right|$, we get the same result given by Newton's law of gravitation, shown in Fig. 3(d). We stress that the different gravitational force lies in that the quantum states of the quantum sphere and of the classical sphere are different.

Assuming that there are $N$ fundamental particles whose wave functions are $\phi_{1}(\boldsymbol{x}, t), \cdots, \phi_{j}(\boldsymbol{x}, t), \cdots$, $\phi_{N}(\boldsymbol{x}, t)$, we can determine the formulas used to calculate the acceleration field due to these $N$ particles. Based on the above special case and the classical limit of Newton's law of gravitation, it is natural to get the following two formulas to calculate the gravitational acceleration of quantum wave packets.

$$
\boldsymbol{a}(\boldsymbol{R}, t)=\frac{2 \pi k_{\mathrm{B}} c}{\hbar} \sum_{j=1}^{N} T_{V j}(\boldsymbol{R}, t) \frac{\nabla_{\boldsymbol{R}} T_{V j}(\boldsymbol{R}, t)}{\left|\nabla_{\boldsymbol{R}} T_{V j}(\boldsymbol{R}, t)\right|},
$$

and

$$
T_{V j}(\boldsymbol{R}, t)=\frac{\hbar G m_{j}}{2 \pi k_{\mathrm{B}} c}\left|\int \mathrm{d}^{3} \boldsymbol{x} \phi_{j}^{*}(\boldsymbol{x}, t) \frac{\boldsymbol{x}-\boldsymbol{R}}{|\boldsymbol{x}-\boldsymbol{R}|^{3}} \phi_{j}(\boldsymbol{x}, t)\right| .
$$

Here, $m_{j}$ is the mass of the $j$ th fundamental particle. The integral on the right-hand side of Eq. (23) is due to the quantum wave packet of the $j$ th fundamental particle; the norm of the vector after calculating this integral indicates that $T_{V j}$ is a scalar field larger than zero. It is easy to show that if all $N$ particles are highly localized classical particles, we obtain Newton's law of gravity $\boldsymbol{a}(\boldsymbol{R})=-\sum_{j} G m_{j}\left(\boldsymbol{R}-\boldsymbol{x}_{j}\right) /\left|\boldsymbol{x}_{j}-\boldsymbol{R}\right|^{3}$, where $\boldsymbol{x}_{j}$ is the location of the $j$ th particle.

Of course, without full understanding of the quantum gravitational effect, we do not have rigorous derivations of these two equations. In the current situation, it is almost impossible to provide a rigorous derivation (even the Schrödinger equation is not obtained with a rigorous derivation). Previous analyses of the thermodynamic origin of gravity provide important clues and confinement conditions that allow the derivation of these two equations. In a sense, the key of the present work lies in the determination of the direction of the gravitational force that gives the correct attractive gravitational effect for classical objects. The same rule is applied to the case of quantum wave packets, which predicts a repulsive gravitational effect. In fact, the absolute value of the acceleration based on Eqs. (22) and (23) is the same as the result based on Newton's law of gravity; the only difference lies in the determination of the direction of the acceleration with the free energy of the whole system when the thermodynamic origin of gravity is considered.

\section{Experimental scheme to test the repulsive gravitational effect}

Now, we turn to consider an experimental scheme to test the repulsive gravitational effect using superfluid ${ }^{4} \mathrm{He}$, as shown in Fig. 5. For the sake of simplicity, we consider a sphere full of superfluid ${ }^{4} \mathrm{He}$. There is a hole in this sphere. In this situation, from Eqs. (22) and (23), the gravity acceleration in the hole of the superfluid ${ }^{4} \mathrm{He}$ sphere can be approximated as

$$
\boldsymbol{a}=\frac{4 \pi}{3} G n_{\mathrm{He}} \boldsymbol{R} .
$$

Here, the liquid helium's density is $n_{\mathrm{He}} \approx 550 \mathrm{~kg} / \mathrm{m}^{3}$. From this equation, the anomalous acceleration is $\boldsymbol{a}=$ $1.5 \times 10^{-7} \boldsymbol{R} / \mathrm{s}^{2}$. The gradient of this anomalous acceleration is $1.5 \times 10^{-7} / \mathrm{s}^{2}$. Even if only the condensate component of the superfluid ${ }^{4} \mathrm{He}$ is considered, for a sphere of radius $1 \mathrm{~m}$, the maximum anomalous acceleration due to the condensate component is roughly $10^{-8} \mathrm{~m} / \mathrm{s}^{2}$. It is noteworthy that this value is well within the range of the presently available experimental technique of atom interferometry [31], which is used to measure the acceleration of gravity. Nevertheless, this is a very weak observable effect. Thus, it is unlikely that we will find evidence to verify or falsify this anomalous acceleration without future experiments. Apart from atom interferometry, the measurement of acceleration due to gravity using Bloch oscillation [32-35] for cold atoms in optical lattices, superfluid helium interferometry [36], free-fall absolute gravimeters [37-39], and superconducting gravimeters [40-43] provides other methods to test the abnormal gravitational effect. In particular, the standard deviation of free-fall absolute gravimeters in the present technique is roughly $10^{-8} \mathrm{~m} / \mathrm{s}^{2}$; superconducting gravimeters have achieved sensitivities of one thousandth of one billionth $\left(10^{-12}\right)$ of the Earth's surface gravity.

Because this abnormal gravitational effect could be tested using contrast experiments with superfluid helium and normal helium, and based on the idea that the abnormal gravitational effect is location-dependent, the sensitivities of the gravimeter could be used to test the abnormal gravitational effect. For this reason, it is very promising to test the abnormal gravitational effect in future experiments.

One possible obstacle to a definitive test of the ab- 


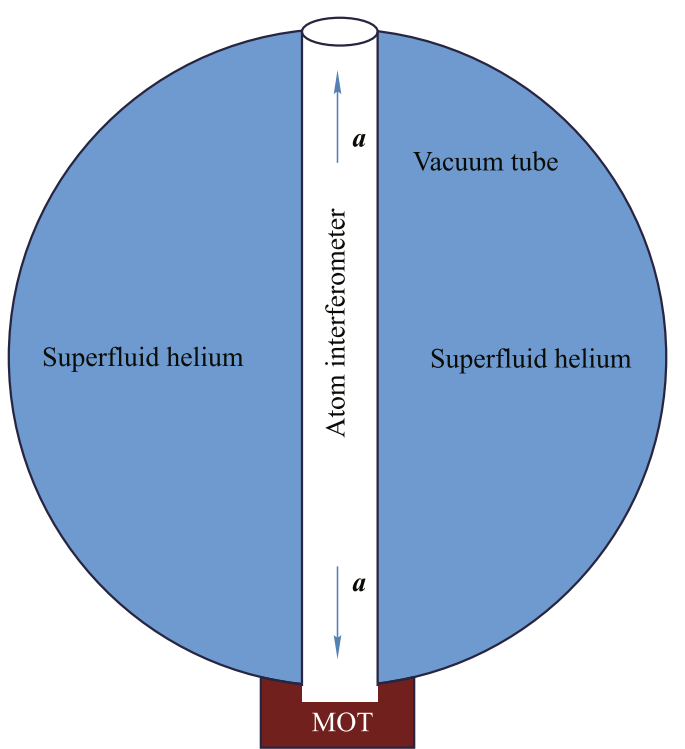

Fig. 5 An experimental scheme to test the abnormal gravitational effect. Various apparatuses measuring the acceleration of gravity are placed in the hole in the superfluid helium sphere. As an example, we consider the application of an atom interferometer, where the vacuum tube of the interference region is placed in the interior of the superfluid helium sphere while the magneto-optical trap (MOT) of the cooling and trapping cold atoms may be placed outside the sphere.

normal quantum gravity effect with a superfluid helium sphere is found in our current understanding of the superfluid behavior of liquid helium. In the ordinary understanding of superfluid helium, the superfluid fraction can reach nearly $100 \%$ while the condensate fraction is roughly $8 \%$. Because of the strong interaction between helium atoms, the liquid helium is a very complex, strongly correlated system. As there are many open questions regarding strongly correlated systems, we cannot exclude the possibility that the wave packets of all helium atoms are localized, although the whole system still exhibits superfluid behavior. This significantly differs from the Bose-Einstein condensate in dilute gases, in which the wave function of the atoms in the condensate is delocalized throughout the condensate. If the wave packets of all of the helium atoms are localized, we cannot observe the repulsive gravitational effect.

\section{Summary and discussion}

In summary, using the thermodynamic origin of gravity, we consider the gravitational effect for classical and quantum objects. We found evidence for a repulsive gravitational effect for quantum wave packets, which can be tested using currently available experimental techniques. Starting from the present phenomenological studies of the gravitational effects of quantum wave packets, it would be useful to consider whether this macroscopic, abnormal gravitational effect could be derived from the microscopic mechanisms of quantum gravity such as superstring with a positive cosmological constant [44], loop quantum gravity and twistor theory [45], etc. Recently, the relation between gravity and thermodynamics has been studied within the framework of loop quantum gravity [46], which provides a possible solution to this problem.

Although the microscopic interaction mechanisms of quantum gravity at the Planck scale are an unsolved problem, the thermodynamics of the macroscopic quantum gravitational effect presented in this work are still meaningful. When statistical mechanics was developed by Boltzmann in 1870, the concept of the atom was still an unrecognized hypothesis (not to mention the collision mechanisms between atoms due to electromagnetic interaction). However, this does not influence the power of statistical mechanics for the description of gas dynamics. On the other hand, the theoretical and experimental advances of statistical mechanics greatly promoted the further understanding of atoms. If the thermodynamic origin of gravity can be verified by future experiments and astronomical observations, investigation into the abnormal gravitational effect will also promote the understanding of quantum gravity at the Planck scale.

Acknowledgements We thank the discussions with Prof. Biao $\mathrm{Wu}$, and his great encouragements. We also thank the great encouragements of Prof. Frank Wilczek, Betsy Devine, and Prof. W. Vincent Liu. This work was supported by the National Key Basic Research and Development Program of China under Grant No. 2011CB921503 and the National Natural Science Foundation of China under Grant Nos. 11175246 and 11334001.

Open Access This article is distributed under the terms of the Creative Commons Attribution License which permits any use, distribution, and reproduction in any medium, provided the original author(s) and the source are credited.

\section{References}

1. S. Hawking, Black hole explosions? Nature 248(5443), 30 (1974)

2. G. Amelino-Camelia, J. Ellis, N. E. Mavromatos, D. V. Nanopoulos, and S. Sarkar, Tests of quantum gravity from observations of $\gamma$-ray bursts, Nature 393(6687), 763 (1998)

3. U. Jacob and T. Piran, Neutrinos from gamma-ray bursts as a tool to explore quantum-gravity-induced Lorentz violation, Nat. Phys. 3(2), 87 (2007)

4. I. Pikovski, M. R. Vanner, M. Aspelmeyer, M. S. Kim, and C. Brukner, Probing Planck-scale physics with quantum optics, Nat. Phys. 8(5), 393 (2012)

5. R. J. Adler, H. Mueller, and M. L. Perl, A terrestrial search 
for dark contents of the vacuum, such as dark energy, using atom interferometry, Int. J. Mod. Phys. A 26(29), 4959 (2011)

6. C. J. Hogan, Measurement of quantum fluctuations in geometry, Phys. Rev. D 77(10), 104031 (2008)

7. C. J. Hogan and M. G. Jackson, Holographic geometry and noise in matrix theory, Phys. Rev. D 79(12), 124009 (2009)

8. T. Jacobson, Thermodynamics of spacetime: The Einstein equation of state, Phys. Rev. Lett. 75(7), 1260 (1995)

9. J. D. Bekenstein, Black holes and entropy, Phys. Rev. D 7(8), 2333 (1973)

10. J. M. Bardeen, B. Carter, and S. W. Hawking, The four laws of black hole mechanics, Commun. Math. Phys. 31(2), 161 (1973)

11. S. W. Hawking, Particle creation by black holes, Commun. Math. Phys. 43(3), 199 (1975)

12. P. C. W. Davies, Scalar production in Schwarzschild and Rindler metrics, J. Phys. A 8(4), 609 (1975)

13. W. G. Unruh, Notes on black-hole evaporation, Phys. Rev. D 14(4), 870 (1976)

14. T. Padmanabhan, Thermodynamical aspects of gravity: New insights, Rep. Prog. Phys. 73(4), 046901 (2010)

15. See, e.g., R. G. Cai, L. M. Cao, and N. Ohta, Friedmann equations from entropic force, Phys. Rev. D 81, 061501(R) (2010)

16. T. Padmanabhan, Surface density of spacetime degrees of freedom from equipartition law in theories of gravity, Phys. Rev. D 81(12), 124040 (2010)

17. F. W. Shu and Y. G. Gong, Equipartition of energy and the first law of thermodynamics at the apparent horizon, Int. J. Mod. Phys. D 20(04), 553 (2011)

18. M. Li and Y. Wang, Quantum UV/IR relations and holographic dark energy from entropic force, Phys. Lett. B 687(23), 243 (2010)

19. T. W. Wang, Coulomb force as an entropic force, Phys. Rev. D 81(10), 104045 (2010)

20. P. Nicolini, Entropic force, noncommutative gravity, and ungravity, Phys. Rev. D 82(4), 044030 (2010)

21. Y. F. Cai and E. N. Saridakis, Inflation in entropic cosmology: Primordial perturbations and non-Gaussianities, Phys. Lett. B 697(4), 280 (2011)

22. R. Banerjee and B. R. Majhi, Statistical origin of gravity, Phys. Rev. D 81(12), 124006 (2010)

23. L. Modesto and A. Randono, Entropic corrections to Newton's law, arXiv:1003.1998 (2010)

24. J. W. Lee, On the origin of entropic gravity and inertia, Found. Phys. 42(9), 1153 (2012)

25. M. A. Santos and I. V. Vancea, Entropic law of force, emergent gravity and the uncertainty principle, Mod. Phys. Lett. A 27, 1250012 (2012), arXiv: 1002.2454

26. M. R. Setare and D. Momeni, Time varying gravitational constant $G$ via entropic force, Commum. Theor. Phys. 56(4), 691 (2011)
27. E. P. Verlinde, On the origin of gravity and the laws of Newton, J. High Energy Phys. 04, 029 (2011)

28. A. G. Riess, A. V. Filippenko, P. Challis, A. Clocchiatti, A. Diercks, P. M. Garnavich, R. L. Gilliland, C. J. Hogan, S. Jha, R. P. Kirshner, B. Leibundgut, M. M. Phillips, D. Reiss, B. P. Schmidt, R. A. Schommer, R. C. Smith, J. Spyromilio, C. Stubbs, N. B. Suntzeff, and J. Tonry, Observational evidence from supernovae for an accelerating universe and a cosmological constant, Astron. J. 116(3), 1009 (1998)

29. S. Perlmutter, G. Aldering, G. Goldhaber, R. A. Knop, P. Nugent, P. G. Castro, S. Deustua, S. Fabbro, A. Goobar, D. E. Groom, I. M. Hook, A. G. Kim, M. Y. Kim, J. C. Lee, N. J. Nunes, R. Pain, C. R. Pennypacker, R. Quimby, C. Lidman, R. S. Ellis, M. Irwin, R. G. McMahon, P. RuizLapuente, N. Walton, B. Schaefer, B. J. Boyle, A. V. Filippenko, T. Matheson, A. S. Fruchter, N. Panagia, H. J. M. Newberg, W. J. Couch, and T. S. C. Project, Measurements of $\Omega$ and $\Lambda$ from 42 high-redshift supernovae, Astrophys. J. $517(2), 565(1999)$

30. P. J. E. Peebles and B. Ratra, The cosmological constant and dark energy, Rev. Mod. Phys. 75(2), 559 (2003)

31. A. D. Cronin, J. Schmiedmayer, and D. E. Pritchard, Optics and interferometry with atoms and molecules, Rev. Mod. Phys. 81(3), 1051 (2009)

32. N. Poli, F. Y. Wang, M. G. Tarallo, A. Alberti, M. Prevedelli, and G. M. Tino, Precision measurement of gravity with cold atoms in an optical lattice and comparison with a classical gravimeter, Phys. Rev. Lett. 106(3), 038501 (2011)

33. P. Cladé, S. Guellati-Khélifa, C. Schwob, F. Nez, L. Julien, and F. Biraben, A promising method for the measurement of the local acceleration of gravity using Bloch oscillations of ultracold atoms in a vertical standing wave, Europhys. Lett. $71(5), 730$ (2005)

34. G. Ferrari, N. Poli, F. Sorrentino, and G. M. Tino, Longlived Bloch oscillations with Bosonic Sr atoms and application to gravity measurement at the micrometer scale, Phys. Rev. Lett. 97(6), 060402 (2006)

35. F. Sorrentino, A. Alberti, G. Ferrari, V. V. Ivanov, N. Poli, M. Schioppo, and G. M. Tino, Quantum sensor for atomsurface interactions below $10 \mu \mathrm{m}$, Phys. Rev. A 79(1), 013409 (2009)

36. E. Hoskinson, Y. Sato, and R. Packard, Superfluid ${ }^{4} \mathrm{He}$ interferometer operating near, Phys. Rev. B 74, 100509(R) (2006)

37. T. M. Niebauer, G. S. Sasagawa, J. E. Faller, R. Hilt, and F. Klopping, A new generation of absolute gravimeters, Metrologia 32(3), 159 (1995)

38. G. d'Agostino, S. Desogus, A. Germak, C. Origlia, D. Quagliotti, G. Berrino, G. Corrado, V. d'Errico, and G. Ricciardi, The new IMGC-02 transportable absolute gravimeter: measurement apparatus and applications in geophysics and volcanology, Ann. Geophys. 51, 39 (2008) 
39. S. Svitlov, P. Maslyk, C. Rothleitner, H. Hu, and L. J. Wang, Comparison of three digital fringe signal processing methods in a ballistic free-fall absolute gravimeter, Metrologia 47(6), 677 (2010)

40. W. A. Prothero and J. Goodkind, A superconducting gravimeter, Rev. Sci. Instrum. 39(9), 1257 (1968)

41. J. Goodkind, The superconducting gravimeter, Rev. Sci. Instrum. 70(11), 4131 (1999)

42. H. J. Paik, Superconducting tunable-diaphragm transducer for sensitive acceleration measurements, J. Appl. Phys. 47(3), 1168 (1976)

43. M. V. Moody and H. J. Paik, Gauss's law test of gravity at short range, Phys. Rev. Lett. 70(9), 1195 (1993)

44. S. Kachru, R. Kallosh, A. Linde, and S. P. Trivedi, de Sitter vacua in string theory, Phys. Rev. D 68(4), 046005 (2003)

45. R. Penrose, Twistor algebra, J. Math. Phys. 8(2), 345 (1967)

46. L. Smolin, Newtonian gravity in loop quantum gravity, arXiv: 1001.3668 (2010) 